\title{
De l'usage de la violence en politique
}

The Use of Violence in Politics

\section{Laurent Bonelli}

\section{OpenEdition}

\section{Journals}

Édition électronique

URL : http://journals.openedition.org/conflits/18093

DOI : $10.4000 /$ conflits. 18093

ISSN : $1777-5345$

Éditeur :

CCLS - Centre d'études sur les conflits lilberté et sécurité, L'Harmattan

Édition imprimée

Date de publication : 5 septembre 2011

Pagination : 7-16

ISBN : 978-2-296-56086-4

ISSN : 1157-996X

\section{Référence électronique}

Laurent Bonelli, « De l'usage de la violence en politique », Cultures \& Conflits [En ligne], 81-82

Printemps/Été 2011, mis en ligne le 05 septembre 2012, consulté le 30 mars 2021. URL : http:// journals.openedition.org/conflits/18093 ; DOI : https://doi.org/10.4000/conflits.18093 


\title{
De l'usage de la violence en politique
}

\section{Laurent BONELLI}

Laurent Bonelli est maitre de conférences en science politique à l'université de Paris-Onest-Nanterre et membre du groupe d'analyse politique (GAP). Il est corédateur en chef de Cultures \& Conflits.

\begin{abstract}
À en juger par l'activité éditoriale, scientifique et même cinématographique

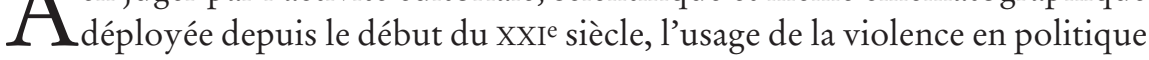
est un thème à la mode. L'activité des groupes armés suscite en effet nombre de témoignages - de ceux qui en ont été membres ou de ceux qui les ont combattus - et de questionnements de la communauté académique, qui cherche tour à tour à comprendre ou à prévenir l'action violente. Le volume des connaissances, comme l'hétérogénéité de leur statut et des contextes dans lesquels elles sont produites, rend difficile une appréhension globale du phénomène.
\end{abstract}

Ainsi, le débat initié en Argentine au début des années 2000 porte sur l'évaluation de l'action des différents mouvements politico-militaires, comme les Montoneros ou le PRT-ERP (Partido Revolucionario de los Trabajadores Ejército Revolucionario del Pueblo), qui s'engagèrent sur la voie de la lutte armée, entre 1958 et $1983^{1}$. En Europe, c'est plutôt l'expérience de groupes allemands (Rote Armee Fraktion), italiens (Brigate Rosse, Prima Linea) ou français (Action directe) des années 1960-1980 qui est mise en scène au cinéma ${ }^{2}$, ou relatée par les protagonistes. D’anciens militants proposent souvent un retour réflexif sur leur expérience, dont ils assument l'échec, mais dont ils tentent de défendre la rationalité sur le moment. "Quand on me presse "Dites-nous pourquoi vous vous êtes trompés" -, je suis prêt à répondre. Mais je ne peux le faire raisonnablement que si l'on regarde les erreurs de tout le

1. Voir Rot G., Los orígenes perdidos de la guerrilla en la Argentina, Buenos Aires, Walter editores 2010.

2. Voir notamment Der Baader Meinhof Komplex (La bande à Baader) de Uli Edel (2008); La Prima linea de Renato de Maria (2009); Ni vieux ni traitres, de Pierre Carles (2005) auxquels on peut ajouter Carlos, de Olivier Assayas (2010) et United Red Army, de Koji Wakamatsu (2008), qui bien que ne portant pas spécifiquement sur l'Europe, explorent la dimension internationaliste de ces mouvements. 
monde et le contexte dans lequel nous étions » indique Mario Moretti, l'un des principaux dirigeants des Brigades Rouges ${ }^{3}$. Pour les policiers qui les traquaient, il s'agit plutôt de dénoncer les protections politiques dont ils ont pu bénéficier, d'évoquer la mémoire de leurs victimes - notamment dans les rangs des forces de l'ordre ou chez leurs informateurs - tout en rappelant leur rôle de l'époque, alors qu'ils sont désormais à la retraite ${ }^{4}$. Quant aux chercheurs, après des écrits pionniers sur les « cycles de violence » consécutifs à l'assèchement des mobilisations ouvrières et étudiantes de la fin des années 1960 5, certains d'entre eux tentent désormais de réconcilier l'analyse de la violence politique et la sociologie de l'action collective ${ }^{6}$. D'autres opèrent plutôt des rapprochements avec leurs collègues spécialisés dans l'étude des groupes nationalistes qui usent de la violence, comme l'Irish Republican Army (IRA), l'Irish National Liberation Army (INLA), l'Ulster Volunteer Force (UVF) en Irlande du nord, Euskadi Ta Askatasuna (ETA) en Espagne ou le Front de Libération Nationale Corse (FLNC) en France. En raison de l'assise sociale de ces groupes clandestins - dont atteste la durée des conflits dans lesquels ils sont engagés -, les auteurs qui s'y intéressent tendent à resituer leur action dans des cadres d'analyse plus larges, qu'ils soient historiques ou « identitaires » 7 .

Pour importants que soient ces débats en Europe et en Amérique, ils restent néanmoins largement occultés par le «terrorisme islamique », sur lequel porte désormais l'essentiel de la littérature. En 2007, Andrew Silke, directeur des terrorism studies à l'Université de East London relevait que depuis les attentats du 11 septembre 2001, un nouveau livre sur le sujet était publié dans le monde anglo-saxon toutes les six heures ${ }^{8}$. Les enquêtes journalistiques, les témoignages plus ou moins vraisemblables de radicaux musulmans « repentis » ou d'anciens agents de renseignement et les analyses stratégiques de think tanks, anglo-saxons surtout, s'ajoutent aux centaines d'articles et d'ouvrages universitaires dressant les caractéristiques de ce qui est présenté comme un

3. Moretti M., Brigate Rosse. Une histoire italienne, Paris, Editions Amsterdam, 2010, p. 350. (Brigate rosse. Una storia italiana, Anabasi 1994).

4. Voir par exemple Pochon J-P., Les stores ronges. Au cour de l'infiltration et de l'arrestation d'action directe (1979-1982), Sainte-Marguerite sur Mer, Edition des équateurs, 2008 ou Savoie S., RG. La traque d'Action directe, Paris, nouveau monde éditions, 2011.

5. Della Porta D., Social Movements, Political Violence and the State. A Comparative Analysis of Italy and Germany, Cambridge, Cambridge University Press, 1995 ; Sommier I., La violence politique et son deuil. L'après 68 en France et en Italie, Rennes, Presses Universitaires de Rennes, 1998. Dans une perspective théorique différente, on peut aussi mentionner Linhardt D., La force de l'État en démocratie. La République fédérale d'Allemagne à l'éprenve de la guérilla urbaine (1967-1982), thèse pour le doctorat en sociologie sous la direction de Bruno Latour, Ecole des Mines de Paris, 2004.

6. L'ouvrage dirigé par Xavier Crettiez et Laurent Mucchielli (Les violences politiques en Europe. Un état des lieux, Paris, La Découverte 2010) est caractéristique de ce mouvement. Voir également Sommier I., Violence et mouvements sociaux. Eléments pour un rapprochement de deux domaines d'études sociologiques, mémoire d'habilitation à diriger les recherches en sociologie, université de Lille 1, 2007.

7. Pour une approche multidimensionnelle du conflit nord-irlandais, voir par exemple Féron E., Abandonner la violence? Comment l'Irlande du Nord sort du conflit, Paris, Payot 2001.

8. "The Rise and rise of terrorism studies", The Guardian, 3 juillet 2007. 
«nouveau terrorisme », du point de vue des relations internationales, de la psychologie, de la philosophie, plus rarement de la sociologie ou de l'anthropologie. Outre les logiques propres aux marchés éditoriaux, l'intérêt des gouvernements et des institutions internationales - fouetté en Europe par les attentats du 11 mars 2004 en Espagne et ceux du 7 juillet 2005 en Grande Bretagne - n'est pas étranger à cet essor : les commandes institutionnelles se sont multipliées. Un groupe d'experts a été constitué en 2006 par la Commission européenne, et ses directions générales « Recherche » et « Justice Liberté et Sécurité » (JLS) ont proposé des études spécifiques. Le Economic and Social Research Council britannique a fait de même.

Si certains travaux sont d'une qualité indéniable, le résultat reste loin d'être intellectuellement satisfaisant. Dans sa revue de la littérature, Silke pointe que $80 \%$ des recherches sont uniquement basées sur des matériaux de seconde main (ouvrages, revues, presse) et que seules $20 \%$ d'entre elles apportent des connaissances nouvelles. De fait, $65 \%$ des articles ne sont que des critiques d'autres publications. Plus grave encore, $1 \%$ seulement des recherches sont basées sur des entretiens et aucune enquête systématique n’a été menée avec des jihadistes 9. La tâche est certes difficile. L'enquête de Farhad Khosrokhavar sur l'islam en prison, qui lui permit d'interroger une quinzaine de militants radicaux incarcérés, en témoigne ${ }^{10}$. Soupçonné d'être un agent des services de renseignements, ou d'être à leur solde, il eut les plus grandes difficultés à créer les « conditions de félicité 11 » qui lui auraient permis de retracer finement les trajectoires sociales, familiales, professionnelles et politiques de ces individus. De fait, il recueillit surtout des proclamations d'innocence, ou, au contraire des discours de revendication de la radicalité, dus à la nature de l'interaction ${ }^{12}$.

Il apparaît assez significatif que l'étude la plus fréquemment citée pour donner les caractéristiques « générales » des « acteurs du jibad» soit celle de Marc Sageman, dans laquelle il dresse le portrait de 172 radicaux islamistes à partir de sources de seconde main. Outre l'absence de groupe contrôle - des individus avec des caractéristiques similaires qui ne passent pas à l'acte - les découpages qu'opère l'auteur demeurent surprenants. Pour lui, les «terroristes [...] musulmans qui sont impliqués dans des mouvements d'insurrection et de guérilla urbaine contre leur propre gouvernement », comme ceux «com-

9. Silke A., "Holy Warriors: Exploring the Psychological Processes of Jihadi Radicalisation", European Journal of Criminology, 5(1), 2008, pp. 99-123

10. Khosrokhavar F., Quand Al-Qaïda parle. Témoignages derrière les barreaux, Paris, Grasset 2006.

11. Goffman E., «La condition de félicité », Actes de la recherche en sciences sociales, $\mathrm{n}^{\circ}$ 64, 1986, pp. 63-78 et n ${ }^{\circ} 65,1986$, pp. 87-98.

12. A l'inverse, on trouvera une rare tentative réussie pour reconstituer sociologiquement la trajectoire de Zacarias Moussaoui, pourtant basée sur des sources secondaires dans Beaud S. et Masclet O., «Un passage à l'acte improbable ? Notes de recherche sur la trajectoire sociale de Zacarias Moussaoui », French Politics Culture and Society, Volume 20, n², été 2002, pp. 159170. 
battant pour la "libération" du Cachemire ou de la Tchétchénie », de même que ceux d'Asie centrale sont exclus de l'échantillon. En revanche, les algériens sont inclus, car en 1995, les attentats menés en France montreraient qu'ils voulaient frapper « l'ennemi lointain » ${ }^{13}$. On appréciera la gymnastique intellectuelle... Plus sérieusement, cette distance au terrain pousse à une surinterprétation des discours publics des acteurs ainsi sélectionnés (le plus souvent de leur traduction anglaise, nombre d'auteurs ne parlant pas les langues dans lesquelles s'expriment les jihadistes), en faisant comme si les raisons d'un conflit pouvaient se déduire de celles dont se revendiquent ses protagonistes. La «nature » d'un conflit (nationaliste, religieux, ou de classe) n'existe que de manière rétrospective, lorsqu'un acteur à conquis une position d'autorité suffisante pour exprimer le point de vue légitime sur celui-ci. La composante religieuse de la guerre de libération algérienne a par exemple été largement gommée après l'indépendance, alors même que les services de renseignements français la considéraient comme essentielle durant son déroulement ${ }^{14}$.

Si l'on excepte quelques spécialistes des sociétés musulmanes, la plupart des chercheurs étudient le radicalisme islamique comme un phénomène en soi et pour soi, en maintenant des frontières étanches avec les autres champs des sciences sociales. Ils font perdurer, sous des habits neufs, une «terroristologie » rappelant la « soviétologie » de naguère, qui prétendait expliquer l'Union soviétique à partir des discours des dirigeants du Parti et de l'interprétation de leur promotion ou de leur relégation. La difficulté de l'enquête n'explique néanmoins pas tout. Le type d'analyse privilégié est en effet à mettre en relation avec les propriétés de ses producteurs, de même qu'avec les positions qu'ils occupent ${ }^{15}$, le plus souvent à la lisière entre le monde académique, les services de renseignement (auxquels ils ont appartenu ou avec lesquels ils entretiennent d'étroites relations), l'expertise publique (dans des commissions nationales ou internationales, des think tanks) et le champ médiatique ${ }^{16}$. La sociologie de cet espace reste à construire rigoureusement, mais son hétérogénéité tout comme son hétéronomie expliquent sans doute la prédominance des approches stratégiques, morales ou centrées sur les individus radicalisés au détriment de celles restituant la violence politique dans son histoire longue. Il s'agit davantage de fournir une aide à la décision politique face à un péril pré-

13. Sageman M., Le vrai visage des terroristes. Psychologie et sociologie des acteurs du djihad, Paris, Denoël, 2005, pp.122-123 (Understanding Terrorist Networks, Philadelphia, Philadelphia University Press, 2004).

14. Voir par exemple Le Dousal R., Commissaire de police en Algérie (1952-1962). Une grenonille dans son puits ne voit qu'un coin du ciel, Paris, Riveneuve éditions, 2011, p. 145.

15. Bourdieu P., Méditations pascaliennes, Paris, Seuil, 2003 (1997), pp. 170-171.

16. La trajectoire de Marc Sageman est de ce point de vue intéressante : il obtient un doctorat de médecine (M.D.) à la NYU School of Medicine en 1979 puis un doctorat de sociologie à la New York University en 1982. Il rejoint ensuite la CIA (Central Intelligence Agency) de 1984 à 1991. De 1987 à 1989, il est à Islamabad et participe aux programmes d'aide au moudjahidines afghans. En 1991, il quitte l'agence et se spécialise en psychiatrie médico-légale. Après les attentats du 11 septembre 2001, il commence à travailler sur les radicaux musulmans. Après la publication de son ouvrage, en 2004, il devient un expert régulièrement consulté par des institutions internationales, les forces de sécurité, les gouvernements et les médias. 
senté comme particulièrement menaçant, que d'être attentif aux dynamiques de conflit.

Dans un texte récent, Donatella Della Porta plaidait pour réinscrire la recherche sur la violence politique dans la tradition des mouvements sociaux, ce qui implique « une prise en compte conjointe des niveaux d'analyse macrocontextuels, organisationnels intermédiaires et micro-individuels. C'est à ces trois niveaux que l'analyse croisée de la violence politique et des mouvements sociaux doit permettre de renouveler l'analyse sur les processus de radicalisation 17 ». On peut la suivre sur ce terrain - riche en terme d'analyse des carrières, des bifurcations, des rétributions, ou de la « disponibilité biographique » des militants, etc. - à condition d'insister sur le caractère relationnel de ces dynamiques. Le passage à la violence n'est presque jamais le fait d'un seul groupe d'acteurs : il résulte de processus dits « d'engrenage » et il est particulièrement significatif que la terminologie de radicalisation utilisée dans les écrits sur le terrorisme occulte celle de l'escalade.

Si l'on étudie les échanges de coups entre acteurs, la relation qui existe entre actions des organisations clandestines, les formes de luttes engagées contre ces organisations, la relation à l'État de droit ou à l'État d'exception, se dégage alors une analyse plus globale de la radicalisation montrant les phénomènes de rivalité mimétique entre les acteurs usant de la violence et ceux cherchant à les réprimer. Les politiques étrangères menées à l'égard de certains pays, les formes de guerre et de coercition, voire de torture, ont un rôle dans la dynamique de radicalisation ${ }^{18}$. La généralisation de mesures de surveillance prises pour lutter contre la radicalisation peut même paradoxalement pousser à la radicalisation. Il est donc nécessaire d'être réflexif sur les politiques mises en œuvre par l'ensemble des acteurs, au lieu de se focaliser uniquement sur les stratégies et tactiques de l'organisation clandestine : c'est ce que l'on appelle «la relation terroriste 19 ».

On l'aura compris, il s'agit de refuser une sorte " d'exceptionnalisme méthodologique »dans l'étude de la violence politique et d'y appliquer les outils ordinaires des sciences sociales. Le passage par la violence en politique n'est pas un «dysfonctionnement », une « subjectivité pathologique 20 » réservée à des fanatiques, à des individus manipulés ou frustrés. Il ne répond pas non plus à des déterminismes sociologiques ou psychologiques, mais s’inscrit

17. Della Porta D., « Mouvements sociaux et violence politique », in Crettiez X. et Mucchielli L., (eds.), Les violences politiques en Europe..., op. cit., p. 288.

18. Bigo D., Bonelli L. et Deltombe Th. (eds.), Au nom du 11 septembre. Les démocraties occidentales à l'éprenve de l'antiterrorisme, Paris, La Découverte, 2008 ; Guittet E. P., Antiterrorisme clandestin, antiterrorisme officiel. Chroniques espagnoles de la coopération en Europe, Outremont, Athéna Editions, 2010.

19. Bigo D. et Hermant D., «La relation terroriste », Études Polémologiques, $\mathrm{n}^{\circ} 47,1988$.

20. Pas plus qu'une «pulsion de mort », que décrit la philosophe Hélène L’Heuillet dans un ouvrage étonnant de normativité. « Les terroristes, explique-t-elle, fanatiques ou embrigadés, mettent leur mort au service de la mort parce qu'ils sont convaincus, au moins le temps de 
dans des configurations sociales, historiques et politiques qu'il faut étudier dans toute leur singularité, en faisant varier les niveaux d'analyse, comme y invite ce numéro de Cultures $\mathcal{E}$ Conflits.

Ainsi, la violence ne surgit-elle pas ex nibilo dans des sociétés relativement pacifiées. Quinze ou vingt ans après la seconde guerre mondiale, de jeunes ouvriers italiens ou des étudiants allemands peuvent croire bien plus que d'autres aux risques de retour de gouvernements fascistes et prendre des armes pour s'y opposer. "Les premières restructurations dans les usines commençaient, la police réprimait de plus en plus durement et les premières bombes ont explosé », raconte Moretti, « avec l'attentat de la piazza Fontana, [...] une seule chose nous paraissait claire : ils étaient en train d'attaquer » 21.

De la même manière, l'apparition d'Action directe en France en 1979 doit beaucoup à l'engagement de certains de ses fondateurs dans la lutte anti-franquiste en Catalogne au début des années 1970. «Nous perpétuons trois décennies de guérilla », écrit avec emphase Jean-Marc Rouillan dans ses mémoires, « nous continuons à tisser le fil ténu nous rattachant à une épopée, à une armée en guenilles et en espadrilles, à l'espoir qui s'écrit en majuscules de poudre et de plomb 22 ».

Dans le cas de l'Allemagne, ce cadre mental vient recouvrir un conflit social de génération marqué par un antagonisme entre des étudiants fortement politisés, mais maintenus en lisière des responsabilités politiques et leurs pères qui les accaparent. Et c'est ici tout l'intérêt du texte de Norbert Elias, jusqu'ici inédit en Français, qui montre à partir de deux configurations différentes (l'Allemagne de Weimar et celle des années 1960-1980) comment ces tensions peuvent se résoudre selon des voies diamétralement opposées. Outre la valeur singulière de la contribution d'un auteur qui a consacré une large part de son œuvre au développement de l'État, à la compréhension d'une violence dirigée contre l'État - pointée par Bernard Lacroix dans son propos introductif cette traduction entend participer à la diffusion des schèmes analytiques qu'il propose, notamment dans la science politique ${ }^{23}$.

leur recrutement, par la négation nihiliste, qui ne promet rien d'autre que de prendre part à la destruction d'un monde haï ». Voir L'Heuillet H., Aux sources du terrorisme. De la petite guerre aux attentats-suicides, Paris, Fayard, 2009, p. 320.

21. Moretti M., Brigate Rosse..., op. cit., p.63. La bombe qui expose le 12 décembre 1969, dans la banque de l'agriculture de Milan faisant seize morts et une centaine de blessés accrédite en effet le risque d'une évolution autoritaire du régime. Ce dont témoigne rétrospectivement Sergio Segio, l'un des fondateurs de Prima Linea : «Les plus avertis d'entre nous accusèrent immédiatement à droite. Mais, l'État était aussi impliqué. Une sombre histoire de services secrets. J'ai grandi avec l'idée qu'ils préparaient un coup d'État, comme en Grèce, ou au Chili. Et qu'ils nous auraient tués. D'ailleurs, ils avaient déjà commencé ». Cité dans La Prima linea de Renato de Maria (2009), réalisé d'après son ouvrage : Miccia corta. Una storia di Prima Linea, Rome, Derive Approdi, 2005.

22. Voir Rouillan J-M., De mémoire (2). Le denil de l'innocence : un jour de septembre 1973 à Barcelone, Marseille, Editions Agone, 2009. 
Ici, on mesure tout l'apport de l'étude des rapports de forces générationnels dans l'analyse des sociétés et des justifications dont ils s'habillent. Par exemple, la «vie de bohème » de la jeunesse bourgeoise et petite-bourgeoise des années 1830, dépeinte par Alfred de Musset dans Confessions d'un enfant $d u$ siècle, comme "une affreuse désespérance " ou « la maladie du siècle » apparaît comme l'une des conséquences du blocage des carrières dans les professions libérales et la haute administration du fait de la présence d'hommes relativement jeunes recrutés sous la Révolution et l'Empire, et du retour des exilés, sous Louis XVIII. Exclus de carrières auxquelles ils pouvaient légitimement prétendre, ils sont "prolongés » dans une adolescence tardive, expliquant la forme particulière que prend le romantisme français à cette période . Dans ces conflits, ce sont les pouvoirs attachés aux différents moments des cycles de vie propres à chaque groupe social qui sont en jeu. Du parricide observable dans les grandes villes italiennes de la Renaissance aux assassinats de la Rote Armee Fraktion, on perçoit le caractère particulièrement heuristique du déplacement du regard au delà des simples actes violents.

L'article d'Antoine Roger s'inscrit également dans cette perspective, lorsqu'il propose d'expliquer les attentats commis par les comités d'action viticoles entre les années 1960 et 1980 et ceux perpétrés par le comité régional d'action viticole à la fin des années 1990 par les fluctuations de l'équilibre des tensions entre les viticulteurs, leurs organisations professionnelles et les autorités publiques en charge de cette question. Les entretiens qu'il a pu mener laissent en effet apparaître comment un répertoire d'action violent peut être mobilisé dans les luttes pour la représentation légitime du groupe viticole, dans une configuration où celle-ci se construit, tout comme dans une configuration où elle est remise en question par le rôle accru joué par les institutions européennes.

Les quatre textes suivants insistent davantage sur le lien entre des militants, leur organisation et la violence 25 . Benjamin Gourisse fournit ainsi une description fine d'une socialisation organisationnelle à la violence dans le mouvement nationaliste en Turquie entre 1975 et 1980. Il montre la formation de véritables commandos, appuyés sur des structures logistiques solides (matérielles et légales) et occupant une place définie dans la division sociale des tâches au sein du parti. Loin d'affaiblir ces groupes, l'accession au gouvernement permet de leur affecter des ressources supplémentaires, qu'elles soient financières ou qu'il s'agisse d'une protection contre la police et la justice. La situation est diamétralement opposée dans l'article de Stéphanie Dechezelles

23. Dans la lignée du travail entrepris dans Garrigou A. et Lacroix B. (dir.), Norbert Elias. La politique et l'histoire, Paris, La Découverte, 1997.

24. Lenoir R., " Objet sociologique et problème social », in Champagne P., Lenoir R., Merllié D. et Pinto L., Initiation à la pratique sociologique, Paris, Dunod, 1996, p. 60.

25. Ces articles ont été rassemblés par Lorenzo Barrault et Caroline Frau, à partir d'une journée d'étude de l'école doctorale de science politique de l'université Paris 1 qui s'est déroulée le 26 septembre 2008. 
portant sur les jeunes de l'Alliance nationale (Alleanza Nazionale) en Italie. Que se passe-t-il lorsque l'usage de la violence de rue - et son exaltation - se dévaluent du fait de la «notabilisation » d'une formation politique ? La participation aux gouvernements de Silvio Berlusconi oblige en effet ce parti, souvent qualifié de « néofasciste » à abandonner ou euphémiser les dimensions les plus visibles de l'engagement violent. Ceci affecte bien sûr les carrières militantes, qui, en fonction de leur ancienneté, ont une inégale capacité à se redéployer dans des combats alternatifs ou dans des activités compensatoires.

Contrairement à des organisations dans lesquelles la violence physique est codifiée et pratiquement institutionnalisée, c'est la découverte de la violence militaire et policière qui façonne la résistance de militants israéliens contre la «barrière de séparation » entre Israël et la Cisjordanie, que décrit Karine Lamarche. L'usage de répertoires d'actions non-violentes, de leur corps comme outil de dénonciation de la brutalité du régime d'occupation amène l'auteure à réfléchir, dans la perspective de Doug Mac Adam, sur l'engagement à risque ${ }^{26}$. La même attention aux trajectoires individuelles se retrouve dans le texte de Marc Milet consacré à Pierre Poujade et Gérard Nicoud, deux dirigeants de mouvements de petits commerçants et artisans, qui eurent volontiers recours à la violence, que ce soit lors de manifestations ou d'actions directes (séquestrations, obstruction des contrôles fiscaux ou plasticages). Il s'agit ici de montrer comment ils ont pu reconvertir cette forme de capital protestataire, le premier pour créer un "système militant néo-patrimonialiste ", le second pour fonder une figure inédite du syndicalisme patronal, celle de médiateur fiscal auprès de l'administration.

Enfin, il a semblé pertinent de présenter dans ce dossier une autre traduction inédite, tirée de l'ouvrage de Enérico García Concha, un militant du mouvement de gauche révolutionnaire chilienne (Movimiento de Izquierda Revolucionaria). Par des anecdotes, l'auteur restitue le mélange singulier d'extraordinaire (l'action armée, la formation militaire, les règles de sécurité) et d'ordinaire (la négligence, la consommation d'alcool) qui constitue la réalité de la clandestinité. Il rend également compte de manière assez précise du contexte politique et social dans lequel la lutte armée peut apparaître comme une option nécessaire.

Outre ce dossier sur la violence en politique, ce numéro de Cultures $\mathcal{E}$ Conflits inaugure une nouvelle rubrique qui reprend celle que les lecteurs d'International Political Sociology connaissent bien. Il s'agit d'un forum dans lequel nous demandons à des collègues et membres du comité de rédaction de prendre position sur une thématique contemporaine afin de pointer ce qui fait débat sur le plan intellectuel, et qui n’a pas nécessairement été éclairé dans les grands media. Il propose un retour sur une « actualité » qui a agité le monde

26. Mac Adam D., "Recruitment to High Risk Activism. The Case of Freedom Summer", American Journal of Sociology, vol. 92, $\mathrm{n}^{\circ} 1,1986$, pp. 64-90. 
politico-médiatique, mais qui en raison de sa temporalité, a laissé place à un autre sujet, sans que l'on puisse se satisfaire des explications communes qui ont été avancées. La situation des Roms en Europe nous a semblé constituer une bonne thématique : boucs émissaires durant quelques semaines, ils sont retournés à l'invisibilité, sans que leur condition ne fasse l'objet - à quelques exceptions près - de réflexion approfondie. 